\title{
Artrodese cervical anterior em três e quatro níveis com dispositivo intersomático não associado à placa cervical
} Anterior cervical arthrodesis for three and four levels using standalone interbody cages without cervical plates Artrodesis cervical anterior en tres y cuatro niveles con dispositivo intersomático no asociado a placa cervical

\author{
Marcel Luiz Benato' \\ Ed Marcelo Zaninell' \\ Xavier Soler i Graells ${ }^{2}$ \\ Marcos Andre Sonagli ${ }^{3}$
}

\section{RESUMO}

Objetivo: avaliar a taxa de consolidação em pacientes submetidos à artrodese cervical anterior de três e quatro níveis utilizando dispositivo intersomático não associado à placa cervical no sexto mês de pós-operatório. Métodos: no período de Novembro de 2005 a Julho de 2008, 20 pacientes foram submetidos ao tratamento cirúrgico proposto. Os critérios de inclusão foram: diagnóstico clínico e por imagem de doença discal degenerativa cervical em três ou quatro níveis; dor axial e/ou irradiada com, no mínimo, seis meses de pós-operatório. O critério de exclusão foi a presença de instabilidade cervical traumática. Foram avaliadas as taxas de consolidação, a presença de sintomas, a taxa de complicações e a posição dos dispositivos intersomáticos (subsidence) após seis meses. Resultados: todos os pacientes obtiveram consolidação em três meses, porém, dois pacientes apresentaram fenômeno de subsi-

\section{ABSTRACT}

Objective: evaluate the rates of fusion of the anterior cervical discectomy and arthrodesis for three and four levels using interbody cages (standalone) without cervical plates six months after post-operative. Methods: from November 2005 to July 2008, 20 patients were treated as proposed. The inclusion criteria were: cervical degenerative disease of three and four levels; axial and/ or irradiated pain at least six months of follow-up. The exclusion criteria were: cervical traumatic instability. The fusion rate, clinical symptoms, rate of complications and the implant position were evaluated six months after post-operative. Results: results were favorable in $100 \%$ of the patients, with residual pain in two cases. Fusion was found in 100\% of the patients, except for two cases with minimum subsidence and fusion in a slight kyphotic position. There were not significant complications.
\end{abstract}

\section{RESUMEN}

Objetivo: evaluar la tasa de consolidación en pacientes sometidos a la artrodesis cervical anterior, de tres y cuatro niveles utilizando dispositivo intersomático no asociado a la placa cervical, después de seis meses de postoperatorio. Métodos: en el período de Noviembre de 2005 a Julio de 2008, 20 pacientes fueron sometidos al tratamiento quirúrgico propuesto. Los criterios de inclusión fueron: diagnóstico clínico y por imagen de enfermedad discal degenerativa cervical en tercer y cuarto niveles; dolor axial y/o irradiada; con mínimo seis meses de postoperatorio. El criterio de exclusión fue la presencia de inestabilidad cervical traumática. Fueron evaluadas las tasas de consolidación, la presencia de síntomas, la tasa de complicaciones y la posición de los dispositivos intersomáticos (subsidente) después de seis meses. Resultados: todos los pacientes obtuvieron consolidación en tres meses, sin embargo, dos pacientes

\footnotetext{
Trabalho realizado pelo Grupo de Cirurgia da Coluna Vertebral do Hospital das Clínicas e Hospital do Trabalhador da Universidade Federal de Paraná - UFPR Curitiba (PR), Brasil.

'Médico Ortopedista; Membro do Grupo de Cirurgia da Coluna Vertebral do Hospital das Clínicas e Hospital do Trabalhador da Universidade Federal do Paraná - UFPR - Curitiba (PR), Brasil.

${ }^{2}$ Mestre; Médico Ortopedista; Chefe do Grupo de Cirurgia da Coluna Vertebral do Hospital das Clínicas e Hospital do Trabalhador da Universidade Federal do Paraná - UFPR - Curitiba (PR), Brasil.

${ }^{3}$ Médico Ortopedista; Especializando do Curso de Cirurgia da Coluna Vertebral do Hospital das Clínicas e do Hospital do Trabalhador da Universidade Federal do
} Paraná - UFPR - Curitiba (PR), Brasil. 
dence, ou seja, migração com consolidação em cifose, sem alterar os resultados clínicos e a consolidação da artrodese após seis meses de pósoperatório. Os pacientes tiveram melhora da dor pré-operatória e apenas três $(15 \%)$ apresentaram dor residual. Não houve complicações maiores. O tempo de hospitalização foi de dois dias. Não foi utilizada imobilização rígida no pós-operatório. Conclusão: obteve-se consolidação com esta técnica em todos os casos. A técnica se mostrou segura e promoveu bons resultados radiológicos e clínicos.

DESCRITORES: Fusão vertebral/ métodos; Artrodese/métodos; Vértebras cervicais/cirurgia; Placas ósseas; Parafusos ósseos; Seguimentos
The discharge of the hospital was performed within two days. Conclusion: The technique is safe and produced good functional and radiological results.

KEYWORDS: Spinal fusion/ methods; Arthrodesis/ methods; Cervical vertebrae/ surgery; Bone plates; Bone screws; Follow-up studies presentaron el fenómeno de subsidente, o sea, migración con consolidación en cifosis, sin alterar los resultados clínicos y la consolidación de la artrodesis a los seis meses de postoperatorio. Los pacientes presentaron mejoría del dolor preoperatorio y solo tres (15\%) presentaron dolor residual. No hubo complicaciones mayores. El tiempo de hospitalización fue de dos días. No fue utilizada inmovilización rígida en el postoperatorio. Conclusión: fue obtenida consolidación con esta técnica en todos los casos. La técnica se mostró segura y promovió buenos resultados radiológicos y clínicos.

\section{DESCRIPTORES: Fusión}

vertebral/métodos; Artrodesis/ métodos; Vértebras cervicales/cirugía; Placas óseas; Tornillos óseos; Estudios de seguimiento

\section{INTRODUÇÃO}

A espondilose cervical acomete cerca de $10 \%$ dos indivíduos aos 25 anos de idade e até $95 \%$ dos indivíduos com mais de 65 anos de idade. Manifesta-se sob a forma de três síndromes álgicas: dor axial, radicular e associação de ambas $^{1}$. O tratamento cirúrgico é uma das formas de tratamento utilizado nessa patologia.

A discectomia cervical anterior com artrodese, conforme descrito por Smith-Robinson, em 1955, para tratamento da espondilose cervical, tem demonstrado alta taxa de sucesso ao longo dos anos. Esse procedimento consiste na descompressão dos níveis acometidos associada à artrodese com bloco de enxerto ósseo tricortical de ilíaco.

Estudos demonstram que a taxa de fusão da artrodese para um único nível, com este procedimento, é de 92 a $100 \%$, e a melhora sintomática está entre 70 e $90 \%$ dos casos. Entretanto, nas discectomias de múltiplos níveis, a taxa de sucesso de fusão diminui conforme aumentam o número de segmentos tratados.

Nas doenças cervicais degenerativas, a literatura cita uma taxa de pseudoartrose entre 10 e $12 \%$ para um único nível, 20 e $27 \%$ para dois níveis e aproximadamente 30 e $56 \%$ em três níveis com esta técnica cirúrgica ${ }^{2}$.

Complicações como a pseudoartrose são responsáveis por $80 \%$ de falhas nas cirurgias de coluna, e o colapso do enxerto autólogo tem sido demonstrado em 20 a $30 \%$ de artrodeses em múltiplos níveis².

A literatura recomenda, portanto, que, na artrodese longa, seja adicionada estabilização com placa cervical anterior ou posterior, para minimizar a taxa de pseudoartrose ${ }^{3}$.

A utilização de placas cervicais está associada à ocorrência de complicações, como quebra ou soltura dos parafusos, lesão esofágica ou lesão medular ou radicular por mau posicionamento dos implantes, além de tempo cirúrgico prolongado e aumento dos custos diretos da cirurgia ${ }^{3}$.

A retirada de enxerto ósseo do ilíaco é associada a diversos tipos de complicações em relação a área doadora, como infecção superficial e profunda, fratura do ilíaco, formação de hematomas, deformidades palpáveis e, eventualmente, visíveis, e até lesão de ramos nervosos locais, que podem desencadear a meralgia parestésica - condição muito dolorosa, de difícil tratamento e que pode prejudicar o resultado clínico da cirurgia cervical ${ }^{4}$. Outra complicação da utilização de blocos de enxerto tricortical de ilíaco é a perda de altura do mesmo, com colapso do espaço discal e consolidação em cifose (subsidence) . $^{5}$.

Diversas técnicas foram desenvolvidas para minimizar a morbidade da área doadora do enxerto. Os maiores avanços dizem respeito aos denominados dispositivos intersomático (cages) ${ }^{2,4}$. Com esse dispositivo, foi possível restaurar o suporte biomecânico imediato, promovendo a osteointegração do enxerto que, em menor quantidade e na forma de osso esponjoso, tem a função de preencher o dispositivo ${ }^{6}$.

Desde 2000, a utilização dos cages cervicais tem se popularizado e, atualmente, é a técnica mais utilizada para a artrodese cervical. Existem cages de diversos materiais, 
porém os mais utilizados são os de titânio e os de PEEK (poli-éter-éter-cetona). Os resultados do procedimento são bons, com melhora significativa dos sintomas de dor. As taxas de artrodese são elevadas - acima de $95 \%$ na maioria das pesquisas científicas ${ }^{2,4}$.

Existem poucos estudos que mostram os resultados com a utilização de cage cervical isolado, seja em PEEK ou titânio, nas discectomias e artrodeses de três ou mais níveis. A maioria deles relata bons resultados, tanto clínicos quanto radiológicos, com taxas de consolidação acima de $90 \%{ }^{7}$.

\section{OBJETIVO}

Relatar a evolução clínica e radiológica de pacientes submetidos à artrodese cervical anterior de três e quatro níveis com cages cervicais preenchidos com enxerto ósseo autólogo, sem a utilização adicional de placa cervical e parafusos.

\section{MÉTODOS}

Foi realizada análise retrospectiva, no período entre Novembro de 2005 e Julho de 2008.

Os critérios de inclusão foram: diagnóstico de doença discal degenerativa cervical em três ou quatro níveis; dor axial, irradiada ou ambas, com, no mínimo, seis meses de pós-operatório. O critério de exclusão foi a presença de instabilidade cervical traumática.

Os pacientes foram avaliados no pré-operatório por radiografias (em incidência ântero-posterior, perfil, oblíquas e perfil em flexão e extensão) e por ressonância magnética. Foi indicado tratamento cirúrgico aos pacientes que não apresentaram melhora dos sintomas com, no mínimo, seis semanas de tratamento conservador (fisioterapia e acupuntura).

Todos os pacientes foram operados por um dos três membros do Grupo de Cirurgia da Coluna Vertebral com a mesma técnica cirúrgica: cervicotomia transversa ou longitudinal esquerda; descompressão até visualização do ligamento longitudinal posterior sem a abertura do mesmo; remoção de osteófitos posteriores, quando presentes; e preservação da placa vertebral óssea dos platôs superiores e inferiores abordados. Os cages utilizados foram de titânio ou PEEK, preenchidos com enxerto ósseo autólogo esponjoso de ilíaco.

Os pacientes utilizaram colar cervical de espuma por duas semanas no pós-operatório. Após esse período, iniciaram fisioterapia visando à reabilitação profissional e ao ganho de amplitude de movimento articular (ADM). O retorno às atividades profissionais ocorreu a partir do terceiro mês pós-operatório.

Os pacientes foram submetidos a reavaliações pósoperatórias em 30, 60, 120 e 180 dias, com exame clínico e radiográfico em incidências ântero-posterior e perfil, sendo realizado perfil dinâmico aos 120 dias de pós-operatório para atestar a consolidação óssea dos níveis abordados, bem como a estabilidade da coluna cervical.
O objetivo primário foi a avaliação das taxas de consolidação da artrodese após seis meses. Os objetivos secundários foram acompanhar a evolução dos sintomas, a taxa de complicações e a posição dos implantes (subsidence) após seis meses de pós-operatório.

\section{RESULTADOS}

A amostra constou de 25 pacientes pré-selecionados, sendo 10 do sexo feminino e 15 do masculino. Após a aplicação dos critérios de inclusão e exclusão, cinco pacientes foram excluídos por apresentarem menos de seis meses de pós-operatório; 20 pacientes permaneceram na pesquisa. Destes, $80 \%$ completaram seguimento pós-operatório de 12 meses. Cinco deles foram abordados em quatro níveis $(25 \%)$ e 15 em três níveis $(75 \%)$. A média de idade foi de 53 anos, variando de 43 e 63 anos. O tempo médio de internação hospitalar foi dois dias.

Após três meses de pós-operatório, observou-se que todos os dispositivos estavam integrados às vértebras, provocando a consolidação - fato este comprovado pelo estudo radiográfico dinâmico em perfil da coluna cervical, bem como pela ausência de sinais de reabsorção óssea dos platôs adjacentes. Foi possível observar adequado crescimento ósseo por meio dos implantes. Essa visualização foi mais fácil nos implantes em PEEK do que nos de titânio. (Figura 1).

Ao final de seis meses, observou-se posicionamento adequado dos implantes em 18 dos 20 casos. (Figura 2).
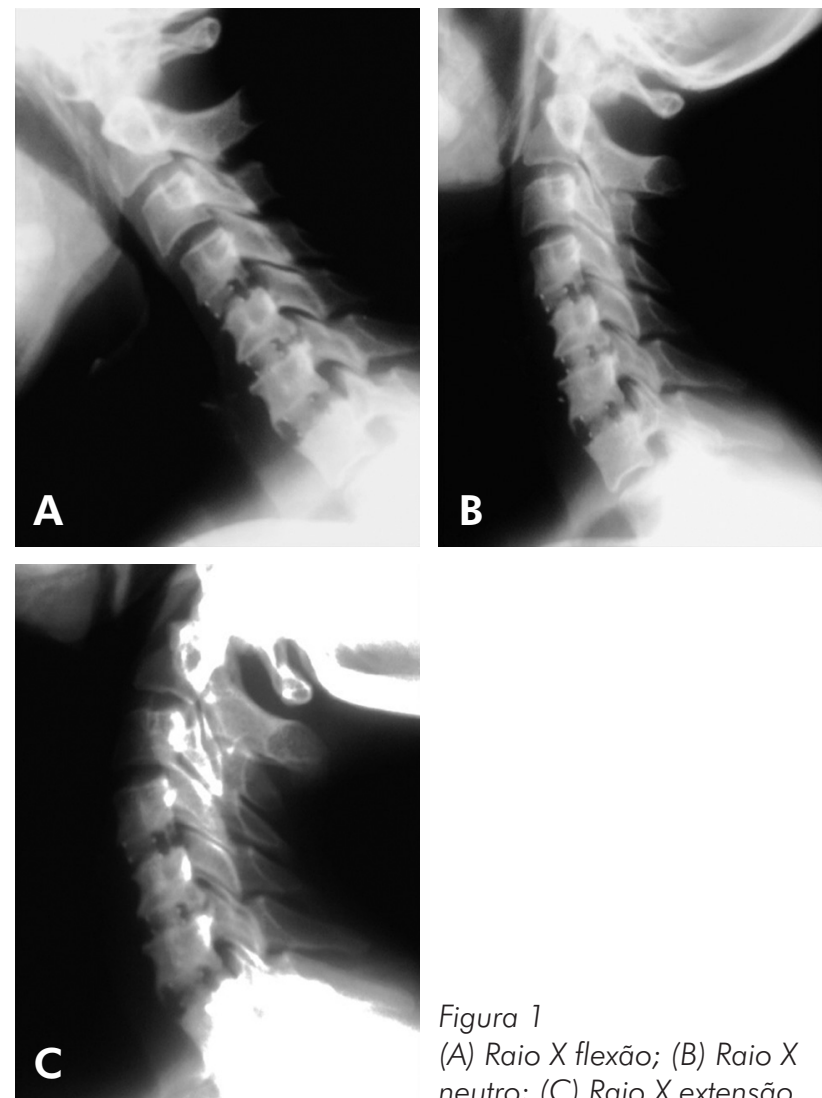

Figura 1

(A) Raio X flexão; (B) Raio X neutro; (C) Raio $X$ extensão. 


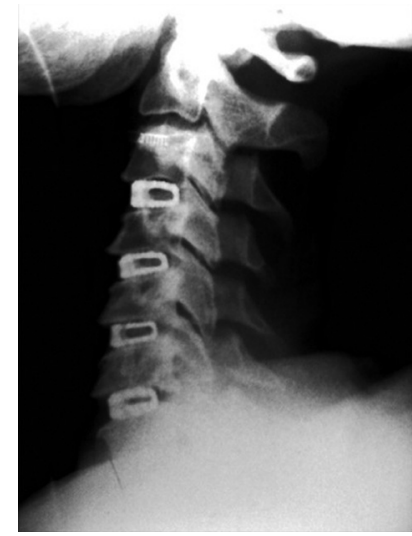

Figura 2

Artrodese cervical quatro níveis, consolidada com cage titânio, após seis meses de pós-operatório.

Dois pacientes tiveram pelo menos um dispositivo com mudança do posicionamento inicial. Em paciente de 62 anos, do sexo feminino, submetida à artrodese em três níveis, os cages sofreram migração parcial para o interior dos corpos vertebrais adjacentes, promovendo consolidação em cifose do segmento. O resultado clínico, entretanto, foi satisfatório. Em outro caso, um paciente de 46 anos, do sexo masculino, foi submetido à artrodese em quatro níveis, e o implante mais caudal migrou parcialmente para o interior do corpo de C7, também causando cifose segmentar, porém sem alterar a boa evolução clínica do caso.

Todos os pacientes apresentaram melhora significativa dos sintomas de dor pré-operatória. Um paciente persistiu com dor irradiada e outro com dor axial, em menor intensidade do que no pré-operatório. Observou-se a ocorrência de três complicações inerentes ao ato cirúrgico: um paciente apresentou disfagia pós-operatória, que foi resolvida em quatro meses após a cirurgia. Dois pacientes apresentaram dor na área doadora do enxerto ósseo do ilíaco, que persistiu por cerca de seis meses após a cirurgia.

Não foi observado nenhum caso de extrusão, anterior ou posterior, dos cages.

\section{DISCUSSÃO}

O desenvolvimento dos cages cervicais foi um grande avanço no tratamento da espondilose cervical. Por sua utilização, foi possível abreviar o tempo de cirurgia, diminuir a necessidade de imobilização pós-operatória e praticamente eliminar as complicações relacionadas ao sítio doador do enxerto ${ }^{2}$.

Ao se analisar a série apresentada, observa-se compatibilidade entre o tipo de doença apresentada e a faixa etária ${ }^{1}$.

A literatura mostra que o seguimento de seis meses é suficiente $^{5}$, uma vez que a maioria dos estudos leva em consideração esse tempo para definir se houve consolidação dos níveis tratados. Na série apresentada, 16 pacientes já apresentavam 12 meses de seguimento; os outros 4 já haviam completado 6 meses.

Dois dias foi o tempo de permanência hospitalar de todos os pacientes da série. Os pacientes foram internados na manhã do dia da cirurgia e receberam alta na tarde do dia seguinte. Isso evidencia a segurança do procedimento, bem como a melhora satisfatória dos sintomas no pós-operatório imediato.

O período de imobilização relativa (com colar cervical de espuma) foi o suficiente para que ocorresse a cicatrização da pele e partes moles. Não foi utilizada a imobilização rígida, sendo que a estabilidade intrínseca dos cages, tanto pelo formato dos implantes com ranhuras craniais e caudais, quanto pelo fato de os cages serem introduzidos sob distração do espaço discal, foram impactados sob pressão.

A primeira avaliação radiográfica confirmava o bom posicionamento dos implantes em 15 dias. Se, porventura, houvesse algum caso de perda do posicionamento, ainda haveria tempo hábil para uma reintervenção. Isso, entretanto, não ocorreu em nenhum paciente.

O método utilizado para determinar a consolidação dos implantes, radiográfico com incidências dinâmicas em flexão e extensão, é o método mais utilizado nos estudos publica$\operatorname{dos}^{8}$. Obteve-se consolidação dos implantes em 100\% dos casos. Mesmo os dois casos de subsidence obtiveram consolidação, ainda que em cifose do segmento. A taxa de consolidação obtida no presente estudo foi semelhante ao estudo de Cho et al. ${ }^{2}$, o qual obteve $100 \%$ de consolidação no grupo tratado com dispositivo cervical isolado em três níveis.

A reabilitação precoce é uma conduta do Grupo de Cirurgia da Coluna Vertebral, que opta pelo menor tempo possível de repouso pós-operatório. Após 15 dias, os pacientes já haviam retirado os pontos e iniciavam a fisioterapia, que foi mantida por 30 sessões, em 100\% dos casos.

A melhora da dor foi significativa. Todos os pacientes apresentaram melhora da dor em algum grau. Mesmo os dois pacientes que permaneceram com dor mostraram-se satisfeitos com o resultado. Os dados da literatura corroboram os encontrados ${ }^{4}$, nos quais se observou que a dor axial é a que persiste com mais frequência após a intervenção.

As complicações do ato cirúrgico per se também foram semelhantes aos da literatura, com dor na área doadora do enxerto em $10 \%$ dos casos e disfagia em 5\% dos casos. A literatura cita taxas de disfagia de até $50 \% \%^{3}$, sendo transitória na grande maioria dos casos.

Explica-se a utilização de implantes de materiais diferentes por uma questão puramente técnica. Em 2005, os cages em PEEK eram raros em nosso meio, motivo pelo qual foram utilizados os implantes em titânio. Atualmente, utilizam-se só cages em PEEK, que, entre outras vantagens, permitem melhor visualização da consolidação, por serem radiotransparentes.

A migração de dois cages (subsidence) observada na série está de acordo com os relatos da literatura ${ }^{5}$. Observase que em um dos casos a paciente já apresentava idade acima de 60 anos e qualidade óssea reduzida, o que favorece a ocorrência de tal fenômeno. Em outro caso, a migração ocorreu no espaço C6-C7, que, segundo a literatura - sem explicação definida, é o local mais acometido pelo subsidence.

A ocorrência da migração (subsidence) com consolidação em cifose em $10 \%$ dos casos não alterou o bom 
resultado clínico. Os estudos mostram que a cifose cervical pode causar compressão secundária da medula sobre a deformidade, estando relacionada a resultados ruins, inclusive com desenvolvimento de mielopatia cervical. No grupo estudado, a cifose foi mínima e em apenas um nível. Não houve nenhum caso de mielopatia na série apresentada.

A ausência de extrusão do implante mostra que sua estabilidade intrínseca é suficiente e segura.

\section{CONCLUSÃO}

Os resultados obtidos mostram que a utilização de dispositivos cervicais isolados (não associados a placas cervicais anteriores) somente preenchidos com enxerto ósseo autólogo em artrodeses cervicais de três e quatro níveis é uma técnica que proporciona bons resultados clínicos e radiográficos, com baixo índice de complicações e altas taxas de consolidação óssea.

\section{REFERÊNCIAS}

1. Connell MD, Wiesel SW. Natural history and pathogenesis of cervical disk disease. Orthop Clin North Am. 1992;23(3):369-80. Review.

2. Cho DY, Lee WY, Sheu PC. Treatment of multilevel cervical fusion with cages. Surg Neurol. 2004;62(5):37885, discussion 385-6.

3. Hee HT, Majd ME, Holt RT, Whitecloud TS 3rd, Pienkowski D. Complications of multilevel cervical corpectomies and reconstruction with titanium cages and anterior plating. J Spinal Disord Tech. 2003;16(1):1-8; discussion 8-9.

4. Rao RD, Currier BL, Albert TJ, Bono CM, Marawar SV, Poelstra KA, et al. Degenerative cervical spondylosis: clinical syndromes, pathogenesis, and management. J Bone Joint Surg Am. 2007;89(6):1360-78. Review.
5. van Jonbergen HP, Spruit M, Anderson PG, Pavlov PW. Anterior cervical interbody fusion with titanium box cage: early radiological assessment of fusion and subsidence. Spine J. 2005;5(6):645-9; discussion 649.

6. Benzel EC. Biomechanics of spine stabilization. New York: Thieme; 2001.

7. Moreland DB, Asch HL, Clabeaux DE, Castiglia GJ, Czajka GA, Lewis PJ, et al. Anterior cervical discectomy and fusion with implantable titanium cage: initial impressions, patient outcomes and comparison to fusion with allograft. Spine J. 2004;4(2):18491; discussion 191. Erratum in: Spine J. 2004;4(3):following table of contents.
8. White AP, Biswas D, Smart LR, Haims A, Grauer JN. Utility of flexion-extension radiographs in evaluating the degenerative cervical spine. Spine. 2007;32(9):975-9.

\section{Correspondência}

Xavier Soler

Central de Serviço de Ortopedia

Hospital de Clínicas de Curitiba da

Universidade Federal do Paraná

Rua General Carneiro, 181, 6 andar

CEP: 80250-180 - Curitiba (PR), Brasil

E-mail: xaviersoler@uol.com.br 